\title{
Qualidade de vida na asma pediátrica: revisão da literatura
}

\author{
Quality of life in asthmatic children: a literature review
}

\author{
Patricia Gomes de Souza', Clemax Couto Sant'Anna ${ }^{2}$, Maria de Fátima B. P. March ${ }^{3}$
}

\section{RESUMO}

Objetivo: Analisar as produções científicas que abordam a temática da qualidade de vida em crianças e adolescentes com asma, objetivando discutir o instrumento de pesquisa Pediatric Asthma Quality of Life Questionnaire (PAQLQ).

Fontes de dados: Revisão não sistemática da literatura incluindo artigos nos idiomas inglês, português e espanhol, a partir das bases de dados Medline, SciELO e Lilacs, no período de 1997 a 2009 . Foram utilizados os termos "quality of life", "asthma pediatrics", "asthma quality of life" e "PAQLQ".

Síntese dos dados: Os 26 artigos encontrados foram organizados em duas seções, a saber: estudos de validação do PAQLQ ( $n=12)$ e estudos de avaliação da qualidade de vida na asma pediátrica usando o questionário PAQLQ $(n=14)$.

Conclusões: A avaliação da qualidade de vida deve ser incorporada à avaliação clínica, uma vez que a doença crônica repercute nas diversas dimensões da vida dos pacientes. $\mathrm{O}$ PAQLQ é de fácil aplicação, reprodutível e constitui-se em instrumento para avaliar a qualidade de vida de crianças e adolescentes com asma.

Palavras-chave: asma; qualidade de vida; criança.

\section{ABSTRACT}

Objective: To analyze scientific articles that discuss the quality of life related to the health of children and adolescents with asthma, focusing the Pediatric Astbma Quality of Life Questionnaire (PAQLQ) evaluation tool.
Data sources: Non-systematic review of the literature including articles in English, Portuguese and Spanish identified via Medline, SciELO and Lilacs, published between 1997 and 2009. The following descriptors were searched: "quality of life", "asthma pediatrics", "astbma quality of life" and "PAQLQ".

Data synthesis: The 26 articles were organized in two sections: studies on validity of PAQLQ $(n=12)$ and the assessment of quality of life in asthmatic children using the PAQLQ ( $\mathrm{n}=14)$.

Conclusions: The assessment of quality of life should be incorporated in clinical evaluations considering that chronic illnesses may affect diverse dimensions of life. The PAQLQ is reliable and easy to use, being an appropriate tool to evaluate quality of life in children and adolescents with asthma.

Key-words: asthma; quality of life; child.

\section{Introdução}

O World Health Organization Quality of Life Group (WHOQOL) definiu qualidade de vida (QV) como "a percepção que o indivíduo tem de si mesmo, da sua posição na vida no contexto de cultura e sistema de valores nos quais ele vive e em relação às suas metas, expectativas e padrões sociais"(1). Para obter uma imagem completa do estado de saúde da criança, devem-se medir os índices clínicos convencionais e a qualidade de vida relacionada à saúde (QVRS) ${ }^{(2)}$.

A asma é uma das principais doenças da infância e da adolescência, cujas repercussões atingem não somente o paciente, mas todo o universo familiar, podendo trazer problemas
Instituição: Universidade Federal do Rio de Janeiro (UFRJ), Rio de Janeiro, RJ, Brasil

${ }^{1}$ Mestranda em Medicina (Clínica Médica) pela UFRJ; Professora Substituta da Universidade Federal Fluminense (UFF), Rio de Janeiro, RJ, Brasil 2Doutor em Medicina (Doenças Infecciosas e Parasitárias) pela UFRJ; Professor Associado da UFRJ, Rio de Janeiro, RJ, Brasil

${ }^{3}$ Doutor em Medicina (Doenças Infecciosas e Parasitárias) pela UFRJ; Professora Adjunta da UFRJ, Rio de Janeiro, RJ, Brasil
Endereço para correspondência:

Patricia Gomes de Souza

Rua Santa Clara, 308/707 - Copacabana

CEP 22041-012 - Rio de Janeiro/RJ

E-mail: patigsouza2000@yahoo.com.br

Conflito de interesse: nada a declarar

Recebido em: 4/8/2010

Aprovado em: 20/5/2011 
complexos e implicações em longo prazo, que irão se traduzir em prejuízo na QV de todo o grupo ${ }^{(3)}$.

Em 1996, Juniper et al ${ }^{(4)}$ desenvolveram o Pediatric Asthma Quality of Life Questionnaire (PAQLQ), validado e publicado na língua inglesa ${ }^{(2)}$. No Brasil, o estudo de tradução e validação para o português foi realizado por La Scala et al em $2005^{(5)}$.

O objetivo do presente estudo foi descrever a QV em crianças e adolescentes com asma revisando a literatura, entre 1997 e 2009, de estudos que utilizaram o questionário PAQLQ como instrumento de avaliação.

\section{Fontes dos dados}

Procedeu-se à busca de artigos indexados em base de dados eletrônicas (Medline, SciELO e Lilacs), utilizando para a busca os descritores: "quality of life", "asthma pediatrics", "asthma quality of life" e "PAQLQ". A busca de artigos no período de 1997 a 2009 ocorreu pelo fato de o PAQLQ ter sido publicado em 1996, procurando-se fazer uma revisão bibliográfica desde sua publicação até a atualidade.

No primeiro levantamento, foram obtidos 522 artigos. Com base na leitura dos resumos, foram excluídos aqueles que não tratavam especificamente de $\mathrm{QV}$ na asma pediátrica por meio do questionário PAQLQ. Foram selecionados 26 artigos: 12 estudos de validação do PAQLQ e 14 de avaliação da QV, sendo organizados nessas duas seções.

\section{PAQLQ}

O questionário PAQLQ foi desenvolvido por Juniper et al no Canadá, que validaram-no, em 1996, na língua inglesa e, posteriormente, em 20 outros idiomas ${ }^{(2,4)}$. O PAQLQ foi desenvolvido para medir a QV em pacientes com asma entre sete e 17 anos de idade. Para sua elaboração, algumas premissas foram levadas em conta e, assim, o instrumento deveria: 1) refletir áreas de função importantes para a criança com asma; 2) avaliar as funções física e emocional; 3) ser reprodutível quando o estado clínico é estável; 4) ser responsivo a mudanças, mesmo que mínimas; 5) ser válido, isto é, ser capaz de medir a $\mathrm{QV}^{(4)}$.

Um questionário contendo 77 itens foi construído e aplicado a cem crianças e adolescentes com asma. Os itens mais citados e apontados como causadores de grande incômodo foram selecionados para compor o PAQLQ.
O questionário final consiste de 23 perguntas divididas em três domínios: sintomas (dez questões), limitação das atividades físicas (cinco questões) e emoções (oito questões). As perguntas são direcionadas às experiências vivenciadas na asma na semana anterior à entrevista. A avaliação da QV é medida por meio de uma escala de respostas de 7 pontos, segundo a qual 1 indica o máximo prejuízo e 7 , nenhum prejuízo ${ }^{(4)}$.

O PAQLQ foi validado transculturalmente em diversos países, incluindo Brasil $^{(5)}$, Turquia ${ }^{(6)}$, Itália ${ }^{(7)}$, Tailândia ${ }^{(8)}$, Holanda ${ }^{(9)}$, Espanha ${ }^{(10)}$, Suécia ${ }^{(11)}$, Singapura ${ }^{(12)}$ entre outros. No Brasil, o estudo de tradução do instrumento para o português (Brasil) e sua validação foi realizado por La Scala et al em 2005(5). Participaram do estudo 56 pacientes entre sete e 17 anos que responderam aos questionários aplicados por um entrevistador ou autopreenchidos. A aplicação do questionário tem duração média de dez minutos e a tradução para a língua portuguesa não modificou sua estrutura original ${ }^{(5)}$.

\section{Estudos de validação do PAQLQ}

O processo do estudo de validação do PAQLQ foi realizado inicialmente pela sua tradução para o idioma do país referente ao estudo e, a seguir, feita a versão (back translation) para o inglês por um tradutor não conhecedor do instrumento ${ }^{(5)}$. Outro fator importante foi a adaptação transcultural do instrumento no país de referência por pessoas que conheçam os guidelines para garantir que o instrumento tenha sido adaptado de forma apropriada para a cultura local e que as propriedades de medidas permaneçam as mesmas do instrumento original ${ }^{(13)}$.

No Brasil, La Scala et al ${ }^{(5)}$ aplicaram o PAQLQ em pacientes com idades entre sete e 17 anos e observaram que crianças com sete ou oito anos de vida não conseguiram compreender o significado de "moderadamente", "frustrado" ou, então, "desconfortado". Para essas crianças o termo foi esclarecido de acordo com o dicionário da língua portuguesa. Em relação às demais idades, não houve nenhum problema no que diz respeito à aplicação do instrumento ${ }^{(5)}$.

A expressão "última semana" foi outro dado levantado como problema de compreensão por crianças na idade entre seis e oito anos na Itália por Ricci et al $l^{(7)}$, assim como por Juniper et $a l^{(4)}$ no Canadá e Elizabeth $e t a l^{(12)} \mathrm{em}$ Singapura. Nesses casos, o termo foi explicado à criança pelos pesquisadores, não sendo permitida a interferência 
dos pais para evitar vieses. Como resultado, o PAQLQ, na versão italiana, foi considerado um instrumento válido para ser usado na prática clínica diária de crianças com asma, entretanto, o PAQLQ não se mostrou capaz de discriminar a QV de crianças com boa função pulmonar ${ }^{(7)}$.

$O$ estudo de validação de Elizabeth et al ${ }^{(12)}$ em Singapura mostrou que o desenho do questionário pode ter contribuído para algumas dificuldades de validação; por exemplo, a linguagem usada para pontuar as respostas. Nesse estudo, crianças desete anos apresentaram dificuldades para diferenciar "bastante incomodado", "mais ou menos incomodado" e "um pouco incomodado", assim como para compreender o conceito "última semana". O PAQLQ foi considerado válido, porém os autores ressaltam ser necessário um estudo ainda maior nessa população e a implementação de algumas modificações, antes que possa ser usado como objeto de pesquisa ou de avaliação clínica.

Badia et al ${ }^{(14)}$, na versão espanhola do PAQLQ, usaram o questionário autopreenchido e notaram que, entre os meninos de sete anos, $80 \%$ autopreencheram o instrumento; mas, entre os meninos de seis anos, apenas $43 \%$ superaram o problema da leitura e puderam completar os questionários. Dificuldades de compreensão do PAQLQ na versão espanhola foram também citadas por Ramirez et al $l^{(15)}$ em crianças de sete e oito anos de idade.

Por outro lado, estudos similares de validação do PAQLQ ${ }^{(6,8-10)}$, com a mesma faixa etária, não observaram tais dificuldades. É o exemplo do PAQLQ na versão holandesa, que apresentou propriedades psicométricas adequadas, boa sensibilidade e uma construção válida, quando analisado em pesquisa transversal e longitudinal ${ }^{(9)}$.

\section{Estudos de avaliação da QV na asma}

Estudos recentes mostraram que pacientes com asma apresentam baixa QV autorrelatada ${ }^{(3,4,16,17)}$. Mesmo sendo pior naqueles com asma grave, os relatos de baixa $\mathrm{QV}$ na asma moderada são consideráveis ${ }^{(3)}$. Qualquer que seja a gravidade da asma, há redução nos domínios físico, psicológico e social da avaliação da QV em geral, com a maioria dos asmáticos apresentando restrições em sua vida e pior status de saúde, comparados a indivíduos sem $\operatorname{asma}^{(4)}$.

No estudo de avaliação da QV com a versão espanhola do PAQLQ ${ }^{(10)}$, observou-se que pacientes com asma leve têm escore mais elevado, sendo a diferença significante para todos os domínios exceto o de atividades. O mesmo foi observado por Yuksell et al $l^{(6)}$ : o escore total e todos os domínios foram mais elevados nas crianças com asma leve, sendo a diferença significante para o escore total e para o domínio "sintomas". Mussaffi et al ${ }^{(18)}$ não encontraram associação entre os escores do PAQLQ e a gravidade da asma; por outro lado, boa correlação foi descrita por Reichenberg e Broberg ${ }^{(11)}$ na Suécia com crianças entre sete e nove anos, e por Guyatt et $a^{(19)} \mathrm{em}$ crianças com idade superior a nove anos.

A duração da asma e sua associação com a QV foi analisada por Yuksell et al ${ }^{(6)}$, sendo observado que o escore total do PAQLQ e de todos os domínios, exceto emoção, foi significantemente maior em crianças cuja duração da doença era de até três anos, comparadas às de duração superior a três anos.

Em uma população pediátrica, Boran et al ${ }^{(20)}$ observaram que as medidas de função pulmonar não apresentaram associação com a QV, à semelhança dos estudos de Mussafi et al ${ }^{(18)}$ e Juniper $e t a^{(4)}$. Em outro estudo, La Scala ${ }^{(21)}$ mostrou não haver correlação entre o volume expiratório forçado no primeiro segundo $\left(\mathrm{VEF}_{1}\right)$ e a média dos domínio, ou com o Escore Clínico de Gravidade (ECG). Pode-se dizer que, nesse estudo, o $\mathrm{VEF}_{1}$ não foi útil para avaliar a $\mathrm{QV}$. Muitos autores têm relatado que o valor pontual do $\mathrm{VEF}_{1}$ reflete fracamente as experiências diárias dos pacientes e não avalia o impacto que a asma causa no indivíduo ${ }^{(4,11,14)}$. Ao contrário do $\mathrm{VEF}_{1}$, as medidas do pico de fluxo expiratório (PFE) tiveram associação significativa com os escores da $\mathrm{QV}^{(4,11,18)}$.

Reichenberg et al ${ }^{(11)}$ e Al-Akour et al $l^{(17)}$ estudaram a relação da QV com a idade dos pacientes com asma e notaram menor índice de QV em crianças mais jovens, semelhante ao trabalho de Boran et al $l^{(20)}$, no qual as crianças com idade mais avançada relataram menos incômodo no domínio sintomas.

Rydstrom $e t$ al $^{(22)}$ associaram a melhor QV com o sexo masculino. Zandieh et a ${ }^{(16)}$ relacionaram a QV e sexo de 113 pacientes asmáticos de sete a 17 anos e observaram pior QV no sexo masculino, semelhante ao encontrado por Nogueira em adolescentes brasileiros ${ }^{(3)}$. Meninos e meninas tiveram uma relação significativa entre gravidade da asma e QV dos três domínios. No sexo masculino, o incômodo foi maior na limitação das atividades, enquanto o baixo índice no domínio "sintomas" foi um dado encontrado nas meninas. Ricci et al ${ }^{(7)}$ relataram que os principais domínios afetados na criança com asma são 
sintomas e atividades. Crianças e adolescentes estão frequentemente preocupados em sofrer uma crise de asma. Tosse e dor no peito causam preocupação neles, além da limitação de exercer atividades que possam exacerbar uma crise de asma e/ou causarem desconforto. A atividade de maior restrição foi a habilidade para correr ${ }^{(11,17,22)}$.

A escolaridade dos pais e a $\mathrm{QV}$ dos pacientes foram avaliados por dois estudos discordantes. No Brasil, Alvim et al $^{(23)}$ estudaram 146 adolescentes que apresentaram índices de QV elevados e cujas mães (72\%) tinham escolaridade superior a quatro anos. Por outro lado, na Espanha, Ramirez et al ${ }^{(15)}$ estudaram 52 pacientes com QV boa, cujas mães (46\%) eram analfabetas. Quanto à escolaridade dos pacientes, a baixa escolaridade teve relação significante $(p<0,05)$ com QV ruim de pacientes portadores de asma ${ }^{(3)}$.

Alguns estudos ${ }^{(11,23)}$ mostraram que fatores socioeconômicos não afetam os escores do PAQLQ. Estudo realizado no Brasil ${ }^{(23)} \mathrm{em}$ adolescentes com asma de escolas públicas não mostrou relação significativa entre as características sociodemográficas e o escore do PAQLQ, exceto para o gênero feminino, que apresentou uma $\mathrm{QV}$ mais baixa. A média do escore do PAQLQ encontrada nesse estudo foi relativamente alta, o que sugere que a $\mathrm{QV}$ avaliada por meio do PAQLQ está mais relacionada à doença do que ao contexto socioeconômico. Por outro lado, investigações diversas mostram associação entre renda familiar e QV em crianças com asma ${ }^{(6,24,25)}$. Van Dellen et al ${ }^{(25)}$ notaram níveis mais baixos de QV em crianças e adolescentes com asma imigrantes, comparados aos não imigrantes, sendo essa diferença relacionada ao nível socioeconômico.

\section{Considerações finais}

A presente revisão mostrou que a $\mathrm{QV}$ de pacientes com asma é influenciada por um conjunto de fatores que interagem: a gravidade dos sintomas, a morbidade, o sexo do paciente, assim como sua capacidade de lidar com certas dificuldades. Fica clara a associação entre um prejuízo na QV e a asma.

O PAQLQ, por se tratar de um questionário recente, em especial no Brasil, onde a validação do ocorreu em 2005 por La Scala et al, ainda foi pouco analisado, sendo escassos os estudos sobre a QV de crianças e adolescentes com asma ${ }^{(5)}$.

O PAQLQ é válido, reprodutível e capaz de detectar mudanças clínicas em crianças e adolescentes com asma $^{(5)}$. Porém, limitações foram levantadas por alguns pesquisadores. Elizabeth et $a^{(12)}$ questionaram a falta de perguntas específicas no PAQLQ para diferentes faixas etárias (crianças e adolescentes) e a não avaliação do impacto social da asma na infância, não gerando informações sobre problemas de absenteísmo escolar, tabagismo na adolescência e/ou uso de anticoncepcionais. De fato, talvez seja um problema do PAQLQ a instituição de um único questionário escrito para idades tão discrepantes ${ }^{(26)}$. Sabendo-se que a compreensão de cada pergunta é diferente em cada idade, perguntas para escolares deveriam ter uma complexidade menor do que as dirigidas aos adolescentes ${ }^{(14)}$. Uma alternativa talvez fosse desenvolver instrumentos específicos para adolescentes ${ }^{(26)}$.

Um ponto também a ser discutido é o fato de o PAQLQ avaliar grupos etários com grande variabilidade de posturas, nível escolar e aspirações ${ }^{(21)}$. Crianças menores (sete ou oito anos de idade) tiveram dificuldade para compreender o significado de "moderadamente", "frustrado" ou "desconfortado", o que poderia ser explicado, mesmo quando alfabetizadas nessa idade, por possuírem um nível cultural ruim ${ }^{(5)}$. Também, pode-se admitir que, pela faixa etária do estudo, os pacientes tenham menor capacidade cognitiva do que os mais velhos. Diante dessa dificuldade, o termo foi esclarecido de acordo com o dicionário da língua local e, mesmo assim, algumas crianças foram excluídas ${ }^{(5)}$. A exclusão de pacientes que não compreendem adequadamente o questionário ou que não sabem ler evitaria que se tivesse uma falsa impressão do instrumento avaliado (viés de informação) ${ }^{(5)}$. Em contrapartida, ambos podem ser considerados erros metodológicos, em se tratando de questionários que, como instrumentos, devem ser autoexplicativos ou, do contrário, os resultados mudariam conforme a interpretação ou a(s) definição(ões) do dicionário utilizado ${ }^{(27)}$. Juniper ${ }^{(28)}$ e Badia $^{(14)}$ aplicaram um teste de leitura padronizado para a população incluída no estudo de validação e adaptação cultural do PAQLQ. No Brasil, porém, além de os educadores questionarem esse tipo de avaliação pelos nossos educadores, não existe um teste de leitura similar ou equivalente aos usados nos estudos anteriormente referidos ${ }^{(27)}$.

Em conclusão, será necessário, no futuro, desenvolver estudos longitudinais que possam avaliar a QV como causa ou efeito de morbidade e gravidade, com o objetivo de auxiliar o desenvolvimento de ações na área da saúde voltadas à garantia de uma boa QV aos pacientes portadores de asma. 


\section{Referências bibliográficas}

1. The Worth Health Organization. Quality of life assessment (WHOQOL): position taper from the World Health Organization. Soc Sci Med 1995;41:1403-9.

2. Juniper EF. How important is quality of life in pediatric asthma? Pediatr Pulmonol 1997;15:17-21.

3. Nogueira KT. Avaliação da qualidade de vida entre adolescentes asmáticos [tese de doutorado]. Rio de Janeiro (RJ): Uerj; 2007.

4. Juniper EF, Guyatt GH, Feeny DH, Ferrie PJ, Griffith LE, Townsend M. Measuring quality of life in children with asthma. Qual Life Res 1996; 5:35-46.

5. La Scala CS, Naspitz CK, Solé D. Adaptation and validation of the Pediatric Asthma Quality of Life Questionnaire (PAQLQ) in Brazilian asthmatic children and adolescents. J Pediatr (Rio J) 2005;81:54-60.

6. Yüksel H, Yilmaz O, Kirmaz C, Eser E. Validity and reliability of the Turkish translation of the Pediatric asthma quality of life questionnaire. Turk J Pediatr 2009;51:154-60.

7. Ricci G, Dondi A, Baldi E, Bendandi B, Giannetti A, Masi M. Use of the Italian version of the pediatric asthma quality of life questionnaire in the daily practice: results of a prospective study. BMC Pediatr 2009;9:30.

8. Poachanukoon O, Visitsunthorn N, Leurmarnkul W, Vichyanond P. Pediatric asthma quality of life questionnaire (PAQLQ): validation among asthmatic children in Thailand. Pediatr Allergy Immunol 2006;17:207-12.

9. Raat H, Bueving HJ, de Jongste JC, Grol MH, Juniper EF, Van der Wouden JC. Responsiveness, longitudinal and cross-sectional construct validity of the pediatric asthma quality of life questionnaire (PAQLQ) in Dutch children with asthma. Qual Life Res 2005;14:265-72.

10. Tauler E, Vilagut G, Grau G, González A, Sánchez E, Figueras G et al. The Spanish version of the paediatric asthma quality of Life questionnaire (PAQLQ): metric characteristics and equivalence with the original version. Qual Life Res 2001;10:81-91.

11. Reichenberg K, Broberg AG. Quality of life in childhood asthma: use of the paediatric asthma quality of life questionnaire in a Swedish sample of children 7 to 9 years old. Acta Paediatr 2000;89:989-95.

12. Elizabeth C, Suzanna S, Tim CF, Chi SL, Mital R, Bee-Wah L. Pediatric asthma quality of life questionnaire: validation in children from Singapore. Asian Pac J Allergy Immunol 1999;17:155-61.

13. Juniper EF. Validated questionnaires should not be modified. Eur Respir J 2009;34:1015-7.

14. Badia LX, García-Hernández G, Cobos N, López-David C, Nocea G,
Roset M et al. Validación de la versión española del pediatric quality of life questionnaire en la valoración de la calidad de vida del niño asmático. Med Clin (Barc) 2001;116:565-72.

15. Narváez CR, Moro PG, Gómez LC, Cos JL, Rallo BL, Rubio CB. Calidad de vida en una población de niños asmáticos. Aten Primaria 2006;38:96-101.

16. Zandieh F, Moin M, Movahedi M. Assessment of quality of life in Iranian asthmatic children, young adults and their caregivers. Iran J Allergy Asthma Immunol 2006;5:79-83.

17. Al-Akour N, Khader YS. Quality of life in Jordanian children with asthma. Int J Nurs Pract 2008;14:418-26.

18. Mussaffi H, Omer R, Prais D, Mei-Zahav M, Weiss-Kasirer T, Botzer Z et al. Computerised paediatric asthma quality of life questionnaires in routine care. Arch Dis Child 2007;92:678-82.

19. Guyatt GH, Juniper EF, Griffith LE, Feeny DH, Ferrie PJ. Children and adult perceptions of childhood asthma. Pediatrics 1997;99:165-8.

20. Boran P, Tokuç G, Pisgin B, Oktem S. Assessment of quality of life in asthmatic Turkish children. Turk J Pediatr 2008;50:18-22.

21. La Scala CS. Qualidade de vida em asmáticos: avaliação de instrumento para uso em crianças e adolescentes. Rev Bras Alergia Imunopatol 2005;28:32-8.

22. Rydström I, Dalheim-Englund AC, Holritz-Rasmussen B, Möller C, Sandman PO. Asthma quality of life for Swedish children. J Clin Nurs 2005;14:739-49.

23. Alvim CG, Picinin IM, Camargos PM, Colosimo E, Lasmar LB, Ibiapina CC et al. Quality of life in asthmatic adolescents: an overall evaluation of disease control. J Asthma 2009;46:186-90.

24. Erickson SR, Munzenberger PJ, Plante MJ, Kirking DM, Hurwitz ME, Vanuya RZ. Influence of sociodemographics on the health-related quality of life of pediatric patients with asthma and their caregivers. J Asthma 2002;39:107-17.

25. Van Dellen QM, Stronks K, Bindels PJ, Öry FG, Bruil J, Van Aalderen WM Health-related quality of life in children with asthma from different ethnic origins. J Asthma 2007;44:125-31.

26. Rutishauser C, Sawyer SM, Bowes G. Quality of life assessment in children and adolescents with asthma. Eur Respir J 1998;12:486-94.

27. Sarria E. Qualidade de vida em crianças asmáticas. J Pediatr (Rio J) 2005;81:268-9.

28. Juniper E, Guyatt G, Feeny D, Griffith L, Ferrie P. Minimun skills required by children to complete health-related quality of life instruments for asthma: comparison of measurement properties. Eur Respir J 1997;10:2286-94. 